\title{
Estudo do efeito de altos teores de cinzas do bagaço de cana-de-açúcar (CBCA) em pastas cimentícias: análise por DRX
}

\author{
Nobre, T. R. S. ${ }^{1 *}$, Santos, T. A. ${ }^{2}$, Argolo R. A. ${ }^{2}$, Ribeiro, D. V. ${ }^{2}$ \\ 1 Faculdades Oswaldo Cruz/Escola Superior de Química, São Paulo, SP, Brasil. \\ 2 Universidade Federal da Bahia/Laboratório de Ensaio de Durabilidade dos Materiais, Salvador, BA, Brasil. \\ *e-mail: thiagorsn@gmail.com
}

\begin{abstract}
Resumo
A busca por materiais alternativos tem sido motivação de diversos estudos, atualmente, devido às questões ambientais e de durabilidade das estruturas de concreto. Assim, a presente pesquisa analisou o efeito da substituição do cimento CP V ARI RS por elevados teores de cinza de bagaço de cana-deaçúcar (CBCA, 20\%, 35\%, 50\% e 70\%) em pastas cimentícias. As pastas foram produzidas com relação água/materiais sólidos igual a 0,5 e a cura foi realizada em uma solução saturada de cal. A análise da reação pozolânica nas pastas foi realizada aos 28 e 91 dias, por meio da difração de raios-X (DRX). Os resultados mostraram que a CBCA apresenta alta atividade pozolânica através da reação da sílica amorfa com a portlandita durante o processo de hidratação. Nas análises de DRX, para as pastas com teores de adição iguais a $35 \%, 50 \%$ e $70 \%$, ocorreu o consumo total de portlandita, confirmando a pozolanicidade da CBCA.
\end{abstract}

Palavras chaves: cinza de bagaço de cana-de-açúcar, portlandita, difração de raios X.

\begin{abstract}
The search for alternative materials has been a motivation of studies, currently, due to environmental and durability of concrete structures issues. Thus, this research analyzes the effect of substitution of cement CP V ARI RS for high contents of sugarcane bagasse ash (SCBA) $(20 \%, 35 \%, 50 \%$ and $70 \%)$ in cement pastes. The pastes were produced with water/dry materials ratio 0,5 and curing was performed in a lime saturated solution. The analysis of the pozzolanic reaction in pastes was performed by 28 and 91 days, through X-ray diffraction. The results showed that SCBA presents high pozzolanic activity through amorphous silica and portlandite reaction during hydration process. In the XRD's analyses, for 35\%, 50\% and $70 \%$ contents, ocurred the portlandite total consumption, confirming SCBA's pozzolanicity.
\end{abstract}

Keywords: sugarcane bagasse ash, portlandite, X-ray diffraction.

\section{Introdução}

As emissões mundiais totais de dióxido de carbono $\left(\mathrm{CO}_{2}\right)$, durante o processo de produção do cimento Portland é de aproximadamente $13 \%$, em relação aos processos industriais [1]. Uma das alternativas para redução do uso de clínquer no cimento e emissão de $\mathrm{CO}_{2}$ é o uso de materiais cimentícios suplementares (MCS). O uso de MCS em substituição parcial ao cimento tem evoluído e materiais alternativos como como as cinzas de casca de arroz, de bagaço de cana- de-açúcar, de castanha de caju e de capim elefante têm sido estudados [2].

O Brasil é o maior produtor mundial de cana-de-açúcar, sendo também, o maior produtor no complexo sucroalcooleiro. Além da importância comercial, a cana de açúcar é um produto agrícola que é capaz de gerar subprodutos com baixo impacto ambiental e alto índice de aproveitamento. Dentre os subprodutos, o bagaço de cana-de-açúcar (BCA) tem sido amplamente utilizado no processo de cogeração de energia, um sistema que viabiliza a geração de energia elétrica para 
a própria usina e para venda às concessionárias, por meio da queima do bagaço.

De acordo com os dados fornecidos pela FIESP/CIESP [3], para cada tonelada de BCA que alimenta o processo de co-geração, são gerados aproximadamente, $25 \mathrm{~kg}$ de cinzas residuais, denominadas cinzas de bagaço de cana-de-açúcar (CBCA).

A cinza proveniente da queima do BCA contém, em sua composição, a sílica, em teores superiores a $60 \%$, em massa. O dióxido de silício $\left(\mathrm{SiO}_{2}\right)$ com estrutura amorfa, dependendo das condições de queima e de sua granulometria, apresentam características químicas e físicas que podem desenvolver atividade pozolânica [4]. Assim, a CBCA pode ser utilizada como uma pozolana em substituição parcial ao cimento Portland.

Estudos têm sido realizados com o intuito de utilizar altos teores de pozolanas em substituição ao cimento em matrizes cimentícias [5 - 8] e, entre estes, estão os resíduos provenientes da queima do bagaço de canade-açúcar.

Entre os principais fatores que influenciam a pozolanicidade da CBCA, é importante destacar: a lavagem prévia do bagaço, a temperatura, o tempo de calcinação e a posterior moagem das cinzas geradas. De acordo com Cook (2006), as condições de queima influenciam no grau de cristalinidade devido à transformação da sílica cristalina em amorfa, além de influenciar na área superficial das partículas [9].

\section{Materiais e métodos}

\subsection{Materiais}

Os materiais utilizados para a produção e caracterização das pastas de cimento foram o bagaço de cana-de-açúcar, cimento Portland CP V ARI RS e água destilada.

O bagaço de cana-de-açúcar (BCA) foi coletado pela empresa União Açucareira, localizada no município Amélia Rodrigues, no Estado da Bahia. O resíduo proveniente da produção de açúcar, é armazenado e estocado no pátio da Usina, ao ar livre. Utilizou-se o cimento Portland CP V ARI RS, da marca Mizu, comercialmente encontrado na cidade de Salvador.

\subsection{Métodos}

\subsubsection{Tratamento térmico do $B C A$}

$\mathrm{O}$ bagaço in natura, coletado na usina foi submetido a um processo de lavagem em água corrente, afim de se retirar as impurezas do material. O bagaço foi seco, em temperatura ambiente e, em seguida, em estufa a 100 $\pm 5^{\circ} \mathrm{C}$ por $24 \mathrm{~h}$.

O bagaço foi submetido a um tratamento térmico na temperatura de $600^{\circ} \mathrm{C}$, pois, de acordo com os resultados de DRX obtidos por Ribeiro e Morelli [10], a cinza calcinada a $600^{\circ} \mathrm{C}$ apresentou um caráter amorfo superior quando comparada com as cinzas calcinadas nas temperaturas de 500 e $600^{\circ} \mathrm{C}$. Para a queima, foi utilizado forno modelo LinnElektro Therm, com taxa de aquecimento de $10^{\circ} \mathrm{C} / \mathrm{min}$ e permanência no patamar de queima por seis horas. Após o fim do processo, foi realizado o resfriamento natural da cinza.

\subsubsection{Caracterização dos materiais e das pastas de cimento}

A distribuição granulométrica para as amostras de cimento e CBCA foi realizada por difração a laser no equipamento CILAS, Particle Size Analyser, modelo CILAS 1180 Liquid, no LACER/UFRGS. A faixa de análise utilizada foi de $0,04 \mu \mathrm{m}$ a $2500 \mu \mathrm{m}$.

Os principais compostos mineralógicos do cimento, da CBCA e das pastas de cimento Portland foram identificados por meio de difração de raios-X (DRX), Utilizou-se um difratômetro D2 Phaser Bruker, com tubo de cobre, varredura de $5^{\circ}$ a $75^{\circ}(2 \theta)$, incremento de $0,02 \%$, funcionando a $10 \mathrm{~mA}$ e $30 \mathrm{kV}$, disponível no LEDMa/UFBA. Assim, para identificação foi utilizado o software DifracEva, da Bruker, o qual possui como banco de dados o Crystallography Open Database (COD).

\subsubsection{Produção das pastas de cimento}

As pastas de referência foram produzidas a partir da mistura de cimento Portland e água (sem CBCA). A seguir, subsituíram-se diferentes teores de cimento por CBCA (20\%, 35\%, 50\% e 70\%, em peso) em misturas com relação água/materiais sólidos igual a 0,5 (em massa), conforme pode ser visualizado na Tabela 1. 
Tabela 1. Proporção de mistura das pastas de referência e com CBCA,

\begin{tabular}{ccccc} 
em massa. & & & & \\
Mistura & $\begin{array}{c}\text { Cimento } \\
(\% \mathrm{~m})\end{array}$ & $\begin{array}{c}\text { CBCA } \\
(\% \mathrm{~m})\end{array}$ & $\begin{array}{c}\text { Reionisada } \\
(\% \mathrm{~m})\end{array}$ & $\begin{array}{c}\text { Relaça/materiais } \\
\text { sólidos }\end{array}$ \\
\hline REF & 100,00 & 0,00 & 50,00 & 0,50 \\
\hline CBC20 & 80,00 & 20,00 & 50,00 & 0,50 \\
\hline CBC35 & 65,00 & 35,00 & 50,00 & 0,50 \\
\hline CBC50 & 50,00 & 50,00 & 50,00 & 0,50 \\
\hline CBC70 & 30,00 & 70,00 & 50,00 & 0,50 \\
\hline
\end{tabular}

A pasta foi, então, colocada em recipientes plásticos de aproximadamente $30 \mathrm{ml}$. Estes corpos de prova foram mantidos em sala climatizada por $24 \mathrm{~h}$ e desmoldados. Após a retirada dos moldes, os corpos de prova foram colocados em cura saturada contendo hidróxido de cálcio para serem analisados aos 28 e 91 dias.

Após o período de cura, os corpos de prova foram triturados e fragmentados e, então, secos a $50^{\circ} \mathrm{C}$, durante $4 \mathrm{~h}$, em estufa. A secagem foi realizada com a finalidade de facilitar o processo de moagem e peneiramento. Em seguida, a pasta foi cominuída com o auxílio de um almofariz com gral de ágata até a granulometria totalmente passante em peneira com abertura de malha de $75 \mu \mathrm{m}$.

\section{Resultados e discussões}

\subsection{Caracterização dos materiais}

$\mathrm{Na}$ Tabela 2 são apresentados os resultados granulométricos do cimento CPV ARI RS. Observa-se que o diâmetro médio de partícula é igual 13,60 $\mu \mathrm{m}$.

Tabela 2. Dados granulométricos percentuais do cimento CPV ARI RS.

\begin{tabular}{lllll} 
Material & $\mathbf{D}_{10}$ & $\mathbf{D}_{50}$ & $\mathbf{D}_{90}$ & $\mathbf{D}_{\text {Médio }}$ \\
\hline $\begin{array}{c}\text { Cimento CP } \\
\text { V ARI RS }\end{array}$ & $1,18 \mu \mathrm{m}$ & $10,92 \mu \mathrm{m}$ & $29,72 \mu \mathrm{m}$ & $13,60 \mu \mathrm{m}$ \\
\hline
\end{tabular}

$\mathrm{Na}$ Tabela 3 são apresentados os resultados granulométricos da CBCA. Observa-se que o diâmetro médio de partícula é igual 36,32 $\mu \mathrm{m}$.

\begin{tabular}{lllll}
\multicolumn{5}{l}{ Tabela 3. Dados granulométricos percentuais da CBCA. } \\
\hline Material & $\mathbf{D}_{10}$ & $\mathbf{D}_{50}$ & $\mathbf{D}_{90}$ & $\mathbf{D}_{\text {Médio }}$ \\
\hline CBCA & $6,73 \mu \mathrm{m}$ & $31,45 \mu \mathrm{m}$ & $73,23 \mu \mathrm{m}$ & $36,32 \mu \mathrm{m}$ \\
\hline
\end{tabular}

A análise do difratograma para a amostra de cimento está apresentada na Figura 1.

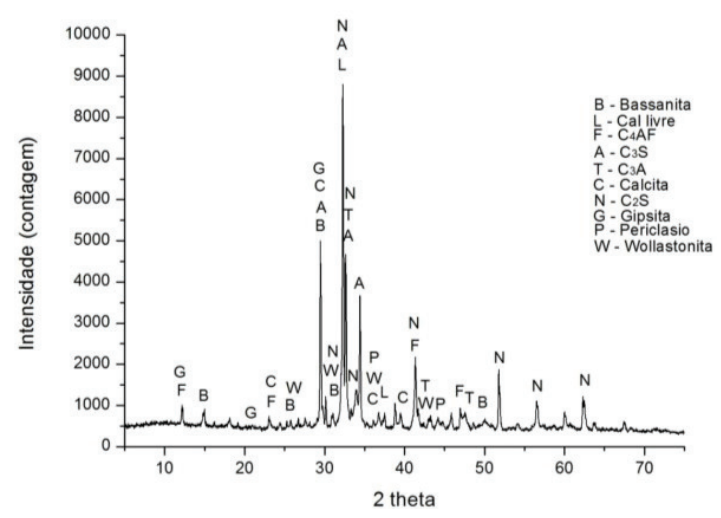

Figura 1. Difratograma do cimento CP V ARI RS anidro.

Podem-se observar picos de larnita (COD: 9012792), alita (COD: 9016125), brownmillerite (COD: 9015720), aluminato tricálcico (COD: 9014359), e calcita (COD: 9016706). Picos de menor intensidade foram observados das seguintes fases: cal livre (COD: 1011094), periclásio (COD: 9007059), wollastonita (COD: 9011913), gipsita (COD: 9013170) e bassanita (COD: 9012209).

No difratograma da CBCA (Figura 2), é possível identificar um halo amorfo indicando fases amorfas, além das cristalinas existentes. Foram identificados picos referentes ao quartzo, uma das formas polimórficas do dióxido de silício (COD: 9012600) e phengite (COD: 9005492).

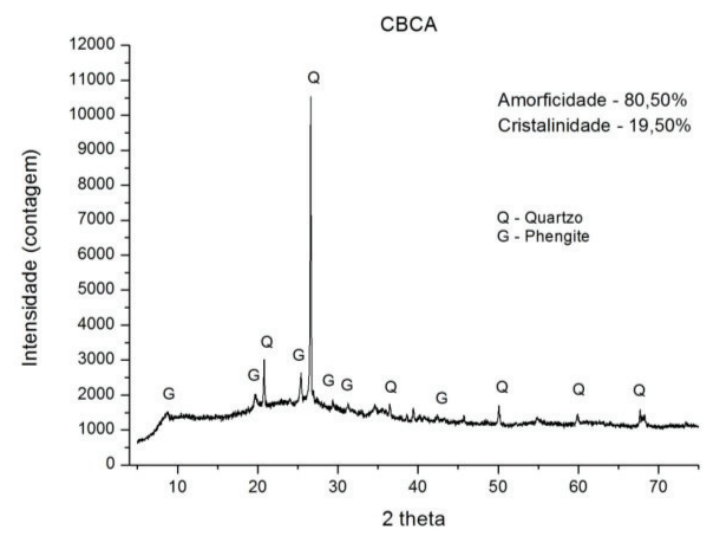

Figura 2. Difratograma da CBCA.

A CBCA caracteriza-se por uma pequena fração cristalina (19,50\%), composta de quartzo e phengite, tendo, em sua composição, majoritariamente fase 
vítrea $(80,50 \%)$. Estes resultados estão em consonância com outros estudos [11 - 13].

Estudos recentes [14] comprovaram que o processo de lavagem da CBCA possibilitou uma redução dos picos cristalinos e a redução do tamanho das partículas médias.

\subsection{Caracterização das pastas de cimento}

A evolução da reação pozolânica, verificada por DRX, das pastas de cimento com diversos teores de CBCA, aos 28 e 91 dias, é apresentada nas Figuras 3 e 4. Para estes ensaios, a varredura foi dividida em dois intervalos $\left(5^{\circ}\right.$ a $25^{\circ}$ e $26^{\circ}$ a $45^{\circ}$, respectivamente).
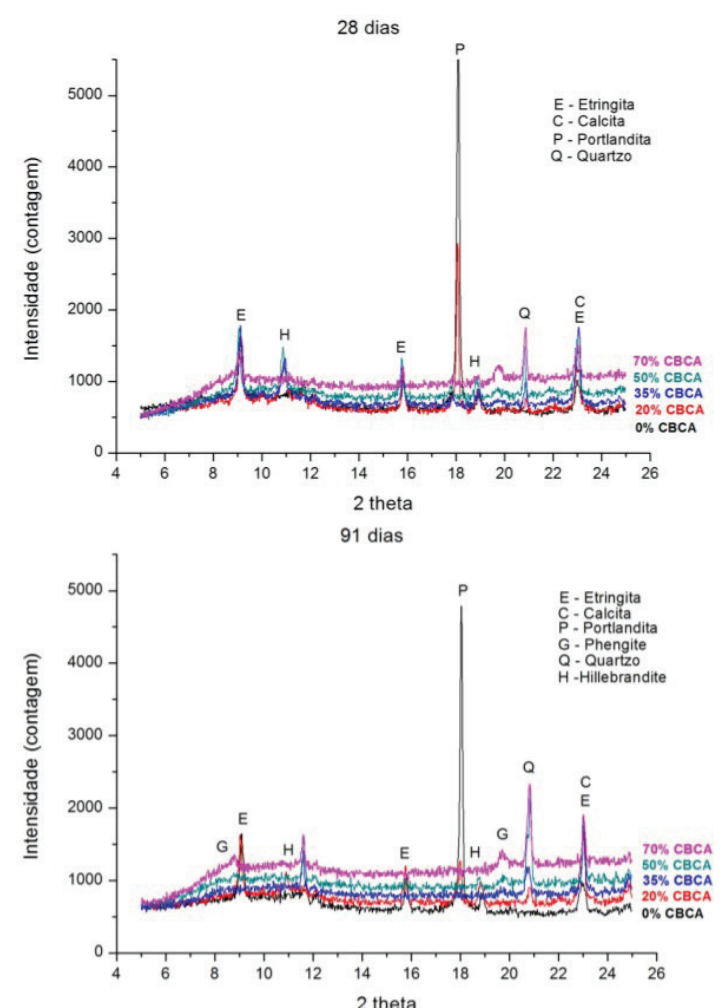

Figura 3. Difratogramas das pastas de cimento de referência $(0 \%)$ e contendo diferentes teores de substituição do cimento pela CBCA $(20 \%, 35 \%, 50 \%$ e $75 \%)$ obtidos aos (A) 28 e (B) 91 dias de idade, para uma varredura $2 \theta$ entre $5^{\circ}$ e $25^{\circ}$.

Pode-se verificar a formação da etringita $\left(9,13^{\circ} 2 \theta\right.$ COD: 9015084) para todos os teores de substituição do cimento pela CBCA, comprovando a reação de hidratação entre os aluminatos e sulfatos presentes no cimento anidro.

Aos 28 dias de hidratação é identificado em 10,95 $(2 \theta)$ a fase hillebrandite, que é uma das fases possíveis para o C-S-H, para os teores $35 \%$ e $50 \%$ de CBCA o que também foi observado em outros estudos [15, 16]. A intensidade do pico secundário da portlandita em $18,11^{\circ}(2 \theta)$, observado na amostra de referência $(0 \%$ CBCA), se reduz na pasta com teor de substituição de $20 \%$, devido ao consumo parcial da portlandita pela fase amorfa da CBCA. No entanto, para as pastas contendo $35 \%, 50 \%$ e $70 \%$ de CBCA não há identificação do pico da portlandita, em função do consumo total desse composto. Pesquisadores [17, 18] ressaltam que a utilização de materiais pozolänicos tem como desvantagem a suscetibilidade à carbonatação. Desta forma, altos teores de CBCA podem provocar um consumo excessivo da portlandita o que pode, consequentemente, interromper as reações pozolânicas. Esse comportamento pode ocorrer devido à redução do volume de cimento e, consequentemente, à redução da portlandita disponível para a reação pozolânica. Com a indisponibilidade da portlandita para a reação, é possível que partículas da CBCA não entrem em contato com a portlandita, ocorrendo a saturação das mesmas, o que reduz a quantidade de produtos hidratados na matriz.

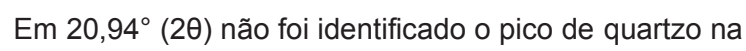
pasta referência, porém, verificou-se a intensificação desta fase com o aumento do teor de CBCA. Este fato ocorre, pois, o quartzo está presente na CBCA, como pode ser constatado no difratograma da Figura 2.

O pico secundário da portlandita $\left(\begin{array}{lll}18,11^{\circ} & 2 \theta\end{array}\right)$, da amostra de referência, aos 91 dias, apresenta-se com menor intensidade, quando comparado com a idade de 28 dias, o que se deve à formação de novas fases da hidratação do cimento ao longo do tempo.

A seguir, são apresentados os difratogramas obtidos em intervalo de varredura (20) entre $26^{\circ}$ e $45^{\circ}$ (Figura 4).

Percebe-se o consumo total do pico principal da portlandita em $34,15^{\circ}(2 \theta)$, aos 28 e 91 dias para pastas com $35 \%$, 50\% e $70 \%$ de CBCA. A atividade pozolânica requer a presença de álcalis, portlandita e $\mathrm{pH}$ elevado para solubilizar a fase vítrea do material pozolânico. Alguns estudos [17, 18] sugerem a adição de cal para manutenção do $\mathrm{pH}$ das matrizes cimentícias e para maior eficiência da atividade pozolânica. 

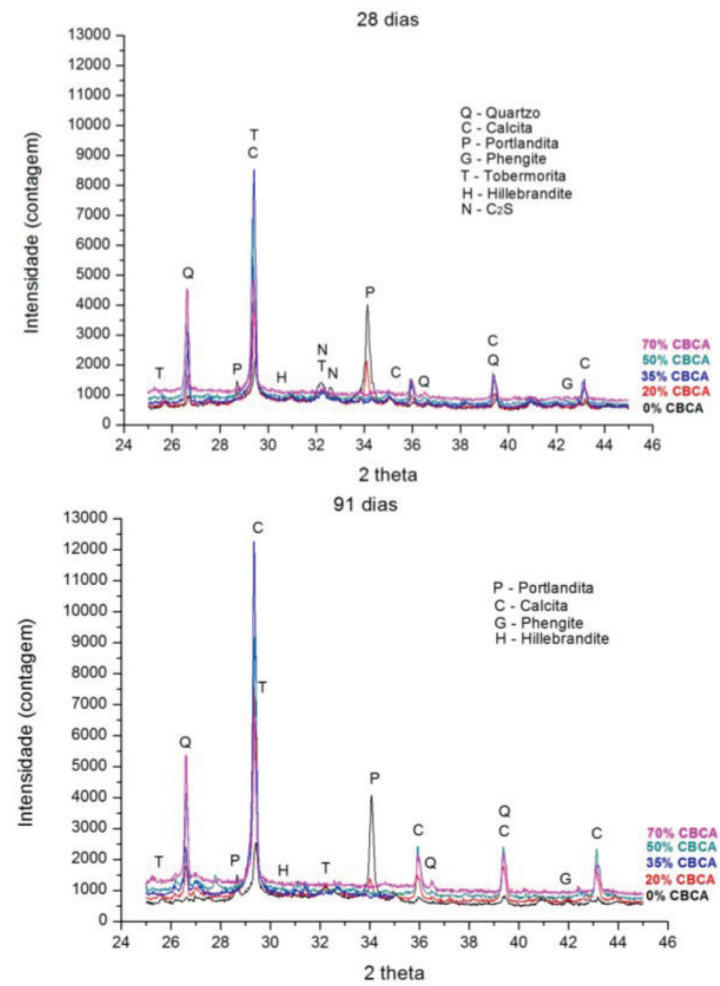

Figura 4. Difratogramas das pastas de cimento de referência $(0 \%)$ e contendo diferentes teores de substituição do cimento pela CBCA ( $20 \%, 35 \%, 50 \%$ e $75 \%$ ) obtidos aos (A) 28 e (B) 91 dias de idade, para uma varredura $2 \theta$ entre $26^{\circ}$ e $45^{\circ}$.

As fases alita, larnite e os aluminatos presentes no cimento anidro diminuíram gradativamente, devido ao aumento do teor de substituição e à evolução da hidratação aos 28 e 91 dias.

O consumo total da portlandita na hidratação do cimento, através da reação pozolânica, é relevante para o desempenho da estrutura de concreto armado. Isto porque o consumo da portlandita resulta na formação de C-S-H secundários na matriz cimentícia, que irão preencher os poros matriz, reduzindo a permeabilidade da pasta de cimento e, consequentemente, a mobilidade de álcalis no concreto. No entanto, a redução da alcalinidade da solução dos poros, devido ao consumo de hidróxido de cálcio, pode favorecer a corrosão, em virtude do processo de despassivação do aço [17].

Dal Molin (2011) ressalta que a redução do pH dos poros da solução e o consumo de hidróxido de cálcio pela reação pozolânica não é um problema quanto à proteção pelo meio alcalino do concreto às armaduras, pois, o valor crítico do $\mathrm{pH}$ para sua despassivação é igual a 9,4, valor que não é atingido devido ao consumo de hidróxido de cálcio pelas reações pozolânicas. Vale ressaltar que a água dos poros da pasta de cimento é alcalina, devido à presença de álcalis $\left(\mathrm{K}^{+}, \mathrm{Na}^{+}\right.$, entre outros), brucita $\left(\mathrm{Mg}(\mathrm{OH})_{2}\right)$ ou dos cátion $\mathrm{Ca}^{2+}$, os quais contribuem para a manutenção do valor crítico do $\mathrm{pH}$.

Nas Figura $5 \mathrm{~A}$ e $5 \mathrm{~B}$ é destacada a área do difratograma que contém o pico principal do quartzo e da portlandita, respectivamente, aos 91 dias de hidratação.
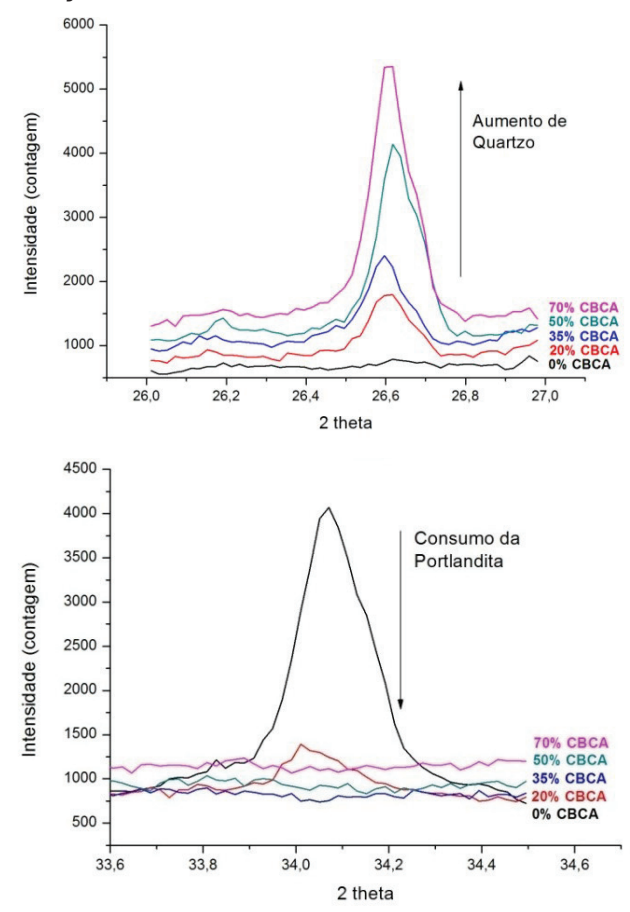

Figura 5. Difratogramas das pastas de cimento de referência $(0 \%)$ e contendo diferentes teores de substituição do cimento pela CBCA $(20 \%, 35 \%, 50 \%$ e $75 \%)$, destacando-se os picos de (A) quarto e (B) portlandita, após 91 dias de hidratação.

A presença de quartzo na CBCA (Figura 5A) não se alterou com a evolução da hidratação. Verifica-se um aumento na intensidade do pico de quartzo com o aumento do teor de substituição do cimento pela CBCA. Analisando-se o pico em $34,15^{\circ}(2 \theta)$, na Figura $5 B$, percebe-se uma redução na intensidade do pico da portlandita, devido à reação desta fase com a sílica amorfa da CBCA (teor 20\%). Acima de 35\% de substituição, não há portlandita disponível nas pastas cimentícias. O uso do alto teor de pozolana em substituição parcial ao cimento, diminui o teor remanescente de portlandita na matriz [19] alterando a distribuição dos poros. As alterações microestruturais decorrentes destes altos teores aumentam a susceptibilidade à carbonatação [20]. 


\section{Conclusões}

A CBCA caracteriza-se por uma pequena fração cristalina (19,50\%), composta de quartzo e phengite, tendo, em sua composição, majoritariamente, fases não cristalinas $(80,50 \%)$. Os resultados desta pesquisa comprovaram que a fase amorfa da CBCA, na presença de portlandita, possibilita a formação de compostos hidráulicos secundários. A reatividade da CBCA foi evidenciada pelo consumo gradual da portlandita ao longo do tempo e com o aumento do teor de substituição da $\mathrm{CBCA}$, o que diminuiu a relação portlandita/cinza, reduzindo, assim, a disponibilidade da portlandita para reagir com a CBCA, como pode ser visto nas misturas com altos teores de substituição (35\%, 50\% e 70\%). Assim sugere-se novos estudos com adição de cal, para substituições elevadas de CBCA.

\section{Agradecimentos}

Os autores agradecem à Fundação Escola Politécnica da Bahia (FEP) pelas bolsas concedidas durante o período da pesquisa.

\section{Referências}

[1] IPCC (Intergovernmental Panel of Climate Change). Chapter 10 - Industry, In "Intergovernmental panel on climate change", Berlin, 2014.

[2] BATTAGIN, A. F. Cimento Portland. In: ISAIA, G. C. (Org.). Concreto: ciência e tecnologia. São Paulo: IBRACON, 2011. p. 185-232.

[3] FIESP/CIESP. Ampliação da oferta de energia através da biomassa (bagaço da cana-de-açúcar). São Paulo: FIESP/CIESP, p. 90, 2001.

[4] CORDEIRO, G. C. et al. Pozzolanic Activity and Filler Effect of Sugar Cane Bagasse Ash in Portland Cement and Lime Mortars. Cement \& Concrete Composites, v. 30, n. 5, p. 410-418, 2008.

[5] HOPPE FILHO, J. Efeitos da Adição de cal Hidratada Sobre a Permeabilidade ao Oxigênio e Absorção Capilar de Concreto Com Altos Teores de Adições Minerais. Dissertação (Mestrado em Engenharia Civil) - Escola de Engenharia, Universidade Federal de Santa Maria, Santa Maria, 2002.

[6] ANDRADE, J. J. O.; LAVARDA, G. B.; NOBRE, T. R. S. The effect of high fly ash content in concrete resistance to acid attack. INCOS 05 - International Conference on Concrete for Structures. University of Coimbra, Coimbra, 7-8 july, 2005.
[7] ISAIA, G. C.; GASTALDINI, A.L.G. Concrete Sustainability With Very High Amount of Fly Ash and Slag. Revista IBRACON de Estruturas e Materiais, v. 2, p. 244-253, 2009.

[8] HOPPE FILHO, J. et al. High-Volume Fly Ash Concrete With and Without Hydrated Lime: chloride diffusion coefficient from accelerated test. Journal of Materials in Civil Engineering, v. 25, n. 3, p. 411-418, 2013.

[9] COOK, D. J. Calcined clay, shale and oil soils. Cement replacement materials, 1986.

[10] RIBEIRO, D. V.; MORELLI, M. R. Effect of Calcination Temperature on the Pozzolanic Activity of Brazilian Sugar Cane Bagasse Ash (SCBA). Materials Research. v. 17. São Carlos, 2014.

[11] CORDEIRO, G. C. Utilização de cinzas ultrafinas do bagaço de cana de açúcar e da casca de arroz como aditivos minerais em concreto. 2006. $445 \mathrm{f}$. Tese (Doutorado em Ciências em Engenharia Civil) Universidade Federal do Rio de Janeiro, Rio de Janeiro. 2006.

[12] BAHURUDEEN, A.; SANTHANAM, M. Influence of different processing methods on the pozzolanic performace of sugar cane bagasse ash. Cement \& Concrete, v. 56, p. 32-45, 2015.

[13] PAYÁ, J. Enhanced conductivity measurement techniques for evaluation of fly ash pozzolanic activity. Cemente and Concrete Research, v. 31, n. 1, p. 41-49, Janeiro 2001.

[14] PEREIRA, A. M. Análise da viabilidade da utilização da cinza do bagaço de cana de açúcar como aglomerante para a produção de matrizes cimentícias. Universidade Estadual de São Paulo. Ilha Solteira. 2014.

[15] SOUZA, P. P. et al. Cement slurries of oil wells under high temperature and pressure: the effects of the use of ceramic waste and silica flour. Brazilian Journal of Petroleum and Gas. v. n.3. p. 105-113. Rio Grande do Norte, 2012.

[16] ANJOS, M. A. S. et al. Cement slurry with silica flour and metakaolin for cementation of oil-wells subject to high temperature. Journal of Materials Science and Engineering, v. 4(12), 2010.

[17] QUARCCIONI, V. A. Influência da cal hidratada nas idades iniciais da hidratação do cimento portland estudo em pasta. Universidade de São Paulo. São Paulo. 2008.

[18] HOPPE FILHO, J. Sistemas cimento, cinza volante e cal hidratada: Mecanismo de hidratação, microestrutura e carbonatação de concreto. Tese. Escola Politécnica, Universidade de São Paulo. São Paulo. 2008.

[19] TAYLOR, H. F. W. The chemistry of cement, Ed. Thomas Telford, Londres, 2a. ed., 1997, 459 p.

[20] HELENE, P. R. L. Contribuição ao estudo da corrosão em armaduras de concreto armado. Tese de Livre Docência, Universidade de São Paulo (USP), São Paulo, 1993. 